JÉRÔME BUZZI

\title{
Ergodicité intrinsèque de produits fibrés d'applications chaotiques unidimensionnelles
}

Bulletin de la S. M. F., tome 126, no 1 (1998), p. 51-77

<http://www.numdam.org/item?id=BSMF_1998_126_1_51_0>

(C) Bulletin de la S. M. F., 1998, tous droits réservés.

L'accès aux archives de la revue «Bulletin de la S. M. F. » (http: //smf.emath.fr/Publications/Bulletin/Presentation.html) implique l'accord avec les conditions générales d'utilisation (http:/www.numdam.org/ conditions). Toute utilisation commerciale ou impression systématique est constitutive d'une infraction pénale. Toute copie ou impression de ce fichier doit contenir la présente mention de copyright.

\section{Numdam}




\section{ERGODICITÉ INTRINSÈQUE DE PRODUITS FIBRÉS D'APPLICATIONS CHAOTIQUES UNIDIMENSIONELLES}

PAR

JÉRÔME BUZZI $\left(^{*}\right)$

RÉSUMÉ. - Nous considérons de petites perturbations fibrées de produits directs d'applications unidimensionelles $C^{\infty}$ d'entropie non-nulle. Nous montrons que ces systèmes dynamiques multidimensionels non-dilatants et non-linéaires ont un nombre non-nul et fini de mesures de probabilité invariantes et ergodiques d'entropie maximale.

La preuve est basée sur la généralisation développée dans [6] du diagramme de Hofbauer. On obtient ainsi l'isomorphisme (au sens de l'entropie) avec une chaîne de Markov topologique dénombrable mais ayant un nombre fini de sous-chaînes irréductibles. Le point essentiel de la preuve est l'estimation d'entropies topologiques par des approximations semi-algébriques et des résultats de semi-continuité de M. Misiurewicz [18] et Y. Yomdin [22].

ABstraCt. - We consider small fibered perturbations of direct products of $C^{\infty}$ interval maps with positive entropy. These non-expanding, non-linear multi-dimensional systems are shown to have a non-zero and finite number of invariant and ergodic probability measures with maximal entropy.

The proof uses a suitable generalization of the Hofbauer diagram developped in [6] which gives isomorphism (in the sense of entropy) with a countable topological Markov chain having a finite number of irreducible sub-chains. The main point of the proof is to estimate topological entropies using approximations by semi-algebraic sets and semi-continuity results of M. Misiurewicz [18] and Y. Yomdin [22].

(*) Texte reçu le 23 juin 1997, révisé le 22 octobre 1997, accepté le 21 novembre 1997. Ce travail a été en partie effectué à l'Université Paris-Sud, Département de Mathématiques, Orsay (France).

J. BuzzI, Laboratoire de Topologie, Université de Bourgogne, CNRS, B.P. 400, 21011 Dijon CEDEX (France). Adresse actuelle : Institut de Mathématiques de Luminy, 163, av. de Luminy, case 930, 13288 Marseille (France). Email : buzzi@iml.univ-mrs.fr

Classification AMS : Primary 58F 11,28D 20.

Mots clés : théorie ergodique, entropie métrique, entropie topologique, ergodicité intrinsèque, applications différentiables, chaîne de Markov topologique, diagramme de Markov, de Hofbauer.

BULlETiN DE LA SOCiÉTÉ MATHÉMATIQUE DE FRANCE $\quad 0037-9484 / 1998 / 51 / \$ 5.00$ (C) Société mathématique de France 


\section{Introduction}

Soient $f_{1}, \ldots, f_{d}$ des applications de l'intervalle $C^{\infty}$ et d'entropie topologique non-nulle. Supposons que les orbites critiques de chaque $f_{i}$ sont non-dégénérées au sens suivant :

$$
f_{i}^{\prime}(c)=0 \Longrightarrow\left\{\begin{array}{l}
f_{i}^{\prime \prime}(c) \neq 0, \\
f_{i}^{\prime}\left(f_{i}^{k}(c)\right) \neq 0, \quad \forall k \geq 1 .
\end{array}\right.
$$

Nous essayons d'analyser la dynamique des applications

$$
F:[0,1]^{d} \longrightarrow[0,1]^{d}
$$

proches dans la topologie $C^{\infty}$ du produit direct $f_{1} \times \cdots \times f_{d}$. Nous nous plaçons du point de vue de la «complexité» : nous nous intéressons aux mesures de probabilités invariantes et ergodiques d'entropie maximale (les mesures dites maximales) et, plus généralement, aux mesures de grande entropie. La première question est celle de l'existence et de la multiplicité des mesures maximales : c'est la question de l'ergodicité intrinsèque due à B. Weiss [21]. Nous allons montrer le :

ThÉORÈme PRINCIPAL. - Soit $F:[0,1]^{d} \rightarrow[0,1]^{d}$ proche de l'application $F_{0}=f_{1} \times \ldots \times f_{d}$ dans la topologie $C^{\infty}$. Supposons que $F$ est fibrée :

$$
F\left(x_{1}, x_{2}, \ldots, x_{d}\right)=\left(\bar{f}_{1}\left(x_{1}\right), \bar{f}_{2}\left(x_{1}, x_{2}\right), \ldots, \bar{f}_{d}\left(x_{1}, \ldots, x_{d}\right)\right)
$$

pour certaines applications $\bar{f}_{i}:[0,1]^{i} \rightarrow[0,1]$. Alors $F$ admet un nombre non-nul et fini de mesures maximales. De plus, chacune de celles-ci est exacte pour un certain itéré de $F$.

Par $« \mu$ exacte pour $F^{p} »$, nous voulons dire qu'on a une partition

$$
[0,1]^{d}=X_{0} \cup \ldots \cup X_{p-1} \quad \text { modulo } \mu
$$

telle que $F\left(X_{i}\right)=X_{i+1}$ (avec $\left.X_{p} \stackrel{\text { def }}{=} X_{0}\right)$ et que $\left(\left.F^{p}\right|_{0}, \mu_{\mid X_{0}}\right)$ est exacte. Ceci implique par exemple que $F^{p} \mid X_{0}$ est mélangeante.

Nous déduisons le théorème principal d'un résultat beaucoup plus précis : nous allons construire un isomorphisme "au sens de l'entropie» (défini ci-dessous) entre $F$ et une chaîne de Markov topologique dont l'ensemble des états est dénombrable mais qui a un nombre fini de souschaînes irréductibles. Cet isomorphisme découle des résultats abstraits de [6]. Les résultats de B. Gurevič [10], [11] sur les chaînes de Markov permettent alors de conclure.

$$
\text { TOME } 126-1998-\mathrm{N}^{\circ} 1
$$




\subsection{Organisation de l'article.}

Nous commençons par rappeler les résultats de [6] concernant l'isomorphisme avec une chaîne de Markov topologique des systèmes inversibles par morceaux (paragraphe 2).

Le paragraphe 3 est consacré à la preuve de la petitesse de l'entropie topologique du bord de la partition d'inversibilité. Nous prouvons que la borne supérieure $h_{\mathrm{top}}^{d-1}(F)$ des entropies topologiques des sous-variétés $C^{\infty}$ de codimension 1 est strictement plus petite que l'entropie totale :

$$
h_{\text {top }}^{d-1}(F)<h_{\text {top }}(F) \text {. }
$$

Remarquons que l'entropie topologique d'une sous-variété (en général noninvariante) est définie ici au sens de Bowen ( $c f$. ci-dessous paragraphe 2).

Le théorème suivant donne cette inégalité dans le cas $F=F_{0}$; on l'étend à un voisinage $C^{\infty}$ de $F_{0}$ par semi-continuité en utilisant des résultats de M. Misiurewicz, Y. Yomdin et M. Gromov.

ThÉORÈme 3.2. - Soient $f_{1}, \ldots, f_{d}:[0,1] \rightarrow[0,1]$ de classe $C^{\infty}$. Supposons que $h_{\mathrm{top}}\left(f_{1}\right) \geq \cdots \geq h_{\mathrm{top}}\left(f_{d}\right)$. Soit $0 \leq k \leq d$. On $a$ :

$$
h_{\text {top }}^{k}\left(f_{1} \times \cdots \times f_{d}\right)=h_{\text {top }}\left(f_{1}\right)+\cdots+h_{\text {top }}\left(f_{k}\right)
$$

Remarquons que ce calcul de $h_{\text {top }}^{d-1}\left(F_{0}\right)$ n'est pas trivial et recourt à l'approximation par des semi-algébriques. Le résultat est d'ailleurs faux si on remplace $C^{\infty}$ par $C^{r}$ avec $r$ fini (voir le contre-exemple de la remarque 3.3 ).

Enfin, le paragraphe 4 conclut la preuve en montrant que la partition de $[0,1]^{d}$ en les composantes connexes de $[0,1]^{d} \backslash\left\{x: \operatorname{det}\left(F^{\prime}(x)\right)=0\right\}$ est une partition d'inversibilité qui sépare presque toutes les orbites au sens de l'entropie. C'est précisément ici que nous utilisons la structure fibrée de $F$ pour faire une récurrence sur la dimension $d$.

Remarque 1.3. - Nous aimerions enlever l'hypothèse de fibration. Remarquons tout d'abord que cette hypothèse est automatiquement satisfaite si on suppose que les applications $f_{i}$ sont fortement dilatantes les unes par rapport aux autres, c'est-à-dire (on remplace $[0,1] \operatorname{par} S^{1}$ ) :

$$
\min _{x \in S^{1}}\left|f_{i}^{\prime}(x)\right|>\max _{x \in S^{1}}\left|f_{i+1}^{\prime}(x)\right|, \quad i=1, \ldots, d-1,
$$

(la dernière application, $f_{d}$, n'est pas forcément dilatante).

C'est un fait connu (voir par exemple [12]).

BULLETIN DE LA SOCIÉTÉ MATHÉMATIQUE DE FRANCE 
De façon plus profonde, nous pensons que c'est l'inégalité entropique (1.2) qui est la condition essentielle dans notre approche et qu'elle devrait suffire à elle seule. Par exemple, nous avons montré [5] qu'elle implique que tous les exposants de Lyapunov des mesures de grande entropie sont strictement positifs et nous espérons pouvoir ainsi récupérer la séparation de presque toutes les orbites. Peut-être n'obtiendrait-on alors qu'une multiplicité dénombrable et non pas finie pour les mesures maximales.

\section{Remerciements.}

J'exprime ma reconnaissance envers $\mathrm{Ph}$. Thieullen qui m'a initié à ce sujet et m'a fournit bien des idées importantes, sans parler de ses encouragements.

\section{Résultats de représentation markovienne}

Nous rappelons des résultats de [6] ce qui nous est nécessaire. Décrivons d'abord le type d'isomorphisme que nous obtiendrons. Rappelons que l'extension naturelle de $(X, f)$, c'est $(\mathcal{X}, \mathcal{F})$ avec :

$$
\begin{gathered}
\mathcal{X}=\left\{x \in X^{\mathbb{Z}} ; \forall p \in \mathbb{Z}, x_{p+1}=f\left(x_{p}\right)\right\}, \\
\mathcal{F}:\left(x_{p}\right)_{p \in \mathbb{Z}} \longmapsto\left(x_{p+1}\right)_{p \in \mathbb{Z}} .
\end{gathered}
$$

Soient $(X, f)$ et $(Y, g)$ des endomorphismes d'espaces mesurables. Notons $(\mathcal{X}, \mathcal{F})$ et $(\mathcal{Y}, \mathcal{G})$ leurs extensions naturelles.

DÉfinition 2.1. - On dit que $(X, f)$ et $(Y, g)$ sont isomorphes au sens de l'entropie, ou simplement $h$-isomorphes, s'il existe des parties invariantes $\mathcal{X}^{\prime} \subset \mathcal{X}, \mathcal{Y}^{\prime} \subset \mathcal{Y}$ telles que :

(1) les systèmes restreints $\left(\mathcal{X}^{\prime}, \mathcal{F}\right)$ et $\left(\mathcal{Y}^{\prime}, \mathcal{G}\right)$ sont conjugués par une bijection bimesurable;

(2) les complémentaires de ces ensembles sont $h$-négligeables, i.e., il existe $H<\sup _{\mu} h_{\mu}(\mathcal{F})$ tel que :

$$
\mu \in \mathrm{M}_{\mathrm{erg}}(\mathcal{X}, \mathcal{F}) \text { et } h_{\mu}(\mathcal{F})>H \Longrightarrow \mu\left(\mathcal{X} \backslash \mathcal{X}^{\prime}\right)=0
$$

et de même pour $\mathcal{Y} \backslash \mathcal{Y}^{\prime}$.

Rappelons qu'une chaîne de Markov topologique est l'ensemble $\Sigma(G)$ des chemins bi-infinis sur un graphe orienté $G$ dénombrable (peut-être fini) avec le décalage $\sigma$ :

$$
\begin{gathered}
\Sigma(G)=\left\{g \in G^{\mathbb{Z}} ; \forall p \in \mathbb{Z}, g_{p} \stackrel{G}{\longrightarrow} g_{p+1}\right\}, \\
\sigma:\left(g_{p}\right)_{p \in \mathbb{Z}} \longmapsto\left(g_{p+1}\right)_{p \in \mathbb{Z}} .
\end{gathered}
$$

TOME $126-1998-\mathrm{N}^{\circ} 1$ 
Les quantités suivantes sont nécessaires à l'énoncé des conditions des théorèmes de représentation markovienne ci-dessous.

Rappelons la définition de l'entropie topologique de Bowen [1] d'une partie (en général non-invariante) de $X$, supposé muni d'une distance $d$. Pour $n>0$, on pose :

$$
d_{n}(x, y)=\max _{0 \leq k<n} d\left(f^{k} x, f^{k} y\right)
$$

Une $(\epsilon, n)$-boule est une boule de rayon $\epsilon>0$ pour la distance $d_{n}$. Un $(\epsilon, n)$-recouvrement est un recouvrement par des $(\epsilon, n)$-boules et le $(\epsilon, n)$ nombre de recouvrement de $S \subset X$ est le nombre minimal de $(\epsilon, n)$-boules nécessaires pour recouvrir $S$; on l'écrit $r(\epsilon, n, S)$.

L'entropie topologique de Bowen de $S \subset X$ est alors :

$$
h_{\mathrm{top}}(S, f)=\lim _{\epsilon \rightarrow 0^{+}} \limsup _{n \rightarrow \infty} \frac{1}{n} \log r(\epsilon, n, S) .
$$

D'après la formule de Katok [14] pour l'entropie métrique, $h_{\mathrm{top}}(S, f)$ majore l'entropie de toute mesure de probabilité invariante et ergodique $\mu$ telle que $\mu(S)>0$.

Pour $S=X$, on obtient l'entropie totale de $f$, notée $h_{\text {top }}(f)$ et, si $f$ est continue et $X$ compact, on a le principe variationnel [8] : « $h_{\text {top }}(f)$ est la borne supérieure des entropies métriques ». On voit donc qu'une partie $S$ est $h$-négligeable dès que $h_{\text {top }}(S, f)<h_{\text {top }}(f)$.

Nous équiperons $F$ d'une partition $P$ de $X=[0,1]^{d}$ qui en fera un système dynamique inversible par morceaux, i.e. :

(1) L'espace $X$ est une partie compacte et propre de $\mathbb{R}^{d}$.

(2) La «partition» $P$ est une collection finie d'ouverts de $\mathbb{R}^{d}$ qui sont non-vides, connexes, deux à deux disjoints et dont l'union est un ouvert dense de $X$. Cette collection $P$ n'est pas une partition au sens ensembliste, mais seulement modulo son bord

$$
\partial P=\bigcup_{A \in P} \partial A
$$

Nous prouverons que ce bord est $h$-négligeable.

(3) L'application $f: X \rightarrow X$ vérifie : chaque restriction $f: \bar{A} \rightarrow \overline{f(A)}$ $(A \in P)$ est un homéomorphisme.

Remarquons qu'une notion plus générale est considérée dans [6]. 
La partition $P$ peut être itérée par $f$ engendrant, pour $n=1,2, \ldots$, l'ensemble des $n$-cylindres

$$
\begin{array}{r}
P^{n}=\left\{\left[A_{0} \ldots A_{n-1}\right] \stackrel{\text { def }}{=} A_{0} \cap f^{-1} \ldots \cap f^{-n+1} A_{n-1} \neq \emptyset ;\right. \\
\left.A_{0}, \ldots, A_{n-1} \in P\right\} .
\end{array}
$$

On écrit $P^{n}(x)$ pour le $n$-cylindre contenant le point $x \in X$, si un tel cylindre existe.

On écrit $P_{n}(x)$ pour la composante connexe de $P^{n}(x)$ contenant $x$.

On dit que la partition $h$-sépare ou sépare $h$-presque toutes les orbites, si pour $h$-presque tout $x \in X$, on a :

$$
\lim _{n \rightarrow \infty} \operatorname{diam}\left(P_{n}(x)\right)=0
$$

(Par $h$-presque tout $x$ on veut dire «tout $x$ en dehors d'un ensemble $h$-négligeable ».)

L'entropie de multiplicité de $(X, P, f)$ est définie par :

$$
h_{\text {mult }}(P, f)=\limsup _{n \rightarrow \infty} \frac{1}{n} \log \operatorname{mult}\left(P^{n}\right)
$$

avec

$$
\operatorname{mult}(Q)=\max _{x \in X} \#\{A \in Q ; x \in \bar{A}\} .
$$

Le diagramme de Markov connexe de $(X, P, f)$ est défini comme

$$
\mathcal{D}=\bigcup_{n \geq 1} \mathcal{D}_{n}
$$

avec

$$
\begin{aligned}
\mathcal{D}_{n}=\left\{f^{k-1}(C) ; C\right. \text { une composante connexe } \\
\text { d'un } k \text {-cylindre avec } k \leq n\} .
\end{aligned}
$$

On a les flèches $A \rightarrow B$ si et seulement si $B$ est une composante connexe de $f(A) \cap Z$ pour un $Z \in P$. Ainsi $\mathcal{D}$ est un graphe orienté dénombrable. Le graphe $\mathcal{D}$ définit une chaîne de Markov topologique $\Sigma(\mathcal{D})$.

ThÉorème 2.2. - Soit $(X, P, f)$ un système dynamique inversible par morceaux. Supposons que :

$$
h_{\mathrm{top}}(\partial P, f)+h_{\text {mult }}(P, f)<h_{\mathrm{top}}(f) .
$$

Supposons aussi que $P$ h-sépare. Alors $(X, f)$ est h-isomorphe à la chaîne de Markov topologique $\Sigma(\mathcal{D})$.

C'est un corollaire immédiat de [6, Main Theorem], à ceci près qu'ici $f$ est définie sur $X$ tout entier. Il suffit donc de vérifier que l'ensemble des orbites qui passent par $\partial P$ est $h$-négligeable. Mais c'est clair d'après l'hypothèse $h_{\text {top }}(\partial P, f)<h_{\text {top }}(f)$ en utilisant la formule de Katok pour l'entropie [14].

$$
\text { TOME } 126-1998-\mathrm{N}^{\circ} 1
$$


ThÉORÈme 2.4. - Sous les mêmes hypothèses, il existe $H<h_{\text {top }}(f)$ et $N<\infty$ tels que chaque sous-chaîne irréductible de $\Sigma(\mathcal{D})$ qui porte des mesures d'entropie au moins $H$ rencontre $\mathcal{D}_{N}$. De plus, $N$ et $H$ ne dépendent de $f$ qu'au travers de la vitesse de convergence des suites définissant $h_{\text {top }}(\partial P, f)$ et $h_{\text {mult }}(P, f)$.

La dernière affirmation veut dire que $N$ et $H$ peuvent être choisis uniformément pour toutes les applications satisfaisant :

$$
\begin{aligned}
& r(\epsilon, n, \partial P) \leq C(\epsilon) \mathrm{e}^{H_{1} n}, \\
& \operatorname{mult}\left(P^{n}\right) \leq C(\epsilon) \mathrm{e}^{H_{2} n},
\end{aligned}
$$

pour tout $n \geq 0, \epsilon>0$, une fonction fixée $\epsilon>0 \mapsto C(\epsilon)<\infty$ et des constantes $H_{1}, H_{2}$ fixées telles que $H_{1}+H_{2}=H<h_{\text {top }}(f)$.

C'est simplement [6, Theorem $\mathrm{C}]$ avec la remarque qui le suit.

\section{Estimations d'entropies}

Le but de ce paragraphe est de montrer que, pour les petites perturbations de produits directs, la borne supérieure des entropies topologiques des sous-variétés $C^{\infty}$ de codimension 1 est strictement plus petite que l'entropie totale. Ceci impliquera que l'entropie topologiqe du bord $\partial P$ est petite.

Nous allons montrer :

THÉorème 3.1. - Soient $f_{1}, \ldots, f_{d}:[0,1] \rightarrow[0,1]$ des applications $C^{\infty}$. Soit $\epsilon>0$. Alors, pour toute application $C^{\infty} F:[0,1]^{d} \rightarrow[0,1]^{d}$ suffisamment proche, dans la topologie $C^{\infty}$, de $f_{1} \times \cdots \times f_{d}$, on a l'inégalité suivante :

$$
h_{\mathrm{top}}(F)>h_{\mathrm{top}}^{d-1}(F)+\min _{i=1, \ldots, d} h_{\mathrm{top}}\left(f_{i}\right)-\epsilon .
$$

En particulier, si toutes les entropies $h_{\mathrm{top}}\left(f_{i}\right)$ sont non-nulles, alors :

$h_{\mathrm{top}}(F)>h_{\mathrm{top}}^{d-1}(F) \quad$ uniformément au voisinage de $f_{1} \times \cdots \times f_{d}$.

Remarquons que cette estimation ne suppose pas $F$ fibrée. Par contre, l'hypothèse de régularité $C^{\infty}$ est cruciale (voir la remarque 3.3 ci-dessous).

\subsection{Calcul pour le produit direct.}

On commence par calculer explicitement

$$
h_{\text {top }}\left(f_{1} \times \cdots \times f_{d}\right) \text { et } h_{\text {top }}^{d-1}\left(f_{1} \times \cdots \times f_{d}\right) .
$$

D'après les définitions, il est trivial que :

$$
h_{\text {top }}\left(f_{1} \times \ldots \times f_{d}\right)=h_{\text {top }}\left(f_{1}\right)+\ldots+h_{\text {top }}\left(f_{d}\right) .
$$


Le calcul de l'entropie topologique de codimension 1 est beaucoup plus délicat, quoique, dans le cas $C^{\infty}$, on obtienne le résultat attendu :

ThÉORÈme 3.2. - Soient $f_{1}, \ldots, f_{d}:[0,1] \rightarrow[0,1] C^{\infty}$ ou bien monotones par morceaux et lipschitziennes. Supposons que $: h_{\mathrm{top}}\left(f_{1}\right) \geq$ $\cdots \geq h_{\text {top }}\left(f_{d}\right)$. Alors, pour tout $k=1,2, \ldots, d$ :

$$
h_{\mathrm{top}}^{k}\left(f_{1} \times \cdots \times f_{d}\right)=h_{\mathrm{top}}\left(f_{1}\right)+\cdots+h_{\mathrm{top}}\left(f_{k}\right) .
$$

Rappelons que $f:[0,1] \rightarrow[0,1]$ est monotone par morceaux s'il existe une partition de $[0,1]$ en un nombre fini d'intervalles tels que la restriction de $f$ à chacun de ces intervalles est un homéomorphisme sur son image.

Remarques 3.3.

(1) Il est trivial de calculer l'entropie des sous-espaces affines parallèles aux axes de coordonnées pour le produit direct. Mais cette estimation ne donne rien pour les perturbations.

(2) L'estimation obtenue pour $h_{\mathrm{top}}^{k}\left(F_{0}\right)$ est celle qu'on attendait. Il est peut-être plus frappant de remarquer que cette estimation est fausse dans le cas $C^{r}, r<\infty$. Nous pensons que ce fait justifie que la démonstration ci-dessous recourre à une technique comme l'approximation par des semialgébriques.

On construit un exemple $C^{r}$ pour $r \geq 1$ un entier arbitraire; c'est une version $C^{r}$ de l'application de Péano. Soit $u: \mathbb{R} \rightarrow \mathbb{R}$ de classe $C^{\infty}$ et de période 1 telle que

$$
u(t)= \begin{cases}0 & \text { pour } 0 \leq t \leq \frac{1}{4} \\ 3 & \text { pour } \frac{1}{2} \leq t \leq \frac{3}{4}\end{cases}
$$

Pour $i=1,2$, soit $\varphi_{i}:[0,1] \rightarrow[0,1]$ l'application de classe $C^{r}$ définie par :

$$
\varphi_{i}(t)=\sum_{k \geq 1} 4^{-3 r k} \frac{4^{3 r}-1}{4-1} u\left(4^{2 k+i-3} t\right) .
$$

Ecrivons $\overrightarrow{0}=0, \overline{2}=3$ et, pour $a \in\{0,2\}^{\mathbb{N}}$,

$$
\left[0 . a_{1} a_{2} \ldots\right]_{4} \stackrel{\text { def }}{=} \sum_{k \geq 1} \frac{a_{k}}{4^{k}} .
$$

On a, les exposants indiquant ici la répétition,

$$
\begin{aligned}
& \varphi_{1}\left(\left[0 . a_{1} a_{2} \ldots\right]_{4}\right)=\left[0 .\left(\overline{a_{1}}\right)^{3 r}\left(\overline{a_{3}}\right)^{3 r} \ldots\right]_{4}, \\
& \varphi_{2}\left(\left[0 . a_{1} a_{2} \ldots\right]_{4}\right)=\left[0 .\left(\overline{a_{2}}\right)^{3 r}\left(\overline{a_{4}}\right)^{3 r} \ldots\right]_{4} .
\end{aligned}
$$

TOME $126-1998-\mathrm{N}^{\circ} 1$ 
Soit $K$ l'ensemble de Cantor

$$
\left\{\left[0 . a_{1} a_{2} \ldots\right]_{4} ; a \in\{0,2\}^{\mathbb{N}}\right\}
$$

Posons

$$
K^{\prime}=\left\{\left[0 .\left(b_{1}\right)^{3 r}\left(b_{2}\right)^{3 r} \ldots\right]_{4} ; b \in\{0,3\}^{\mathbb{N}}\right\} .
$$

Il est clair que l'application

$$
t \longmapsto\left(\varphi_{1}(t), \varphi_{2}(t)\right)
$$

envoie $K \subset[0,1]$ sur $K^{\prime} \times K^{\prime}$.

Il est maintenant facile de définir $g:[-1,2] \rightarrow[-1,2], C^{\infty}$ et monotone par morceaux, de sorte que :

- l'ensemble des points non-errants $\Omega(g)$ soit $K^{\prime} \cup\left\{-\frac{1}{2}\right\}$, avec $-\frac{1}{2}$ un point fixe attractif;

- $h_{\text {top }}(g)>0$ et $g^{(s)}(-1)=0$ pour tout $s \geq 0$.

Soient $f_{i}:[-2,2] \rightarrow[-2,2]$ pour $i=1,2$ définies par

$$
f_{i \mid-1,2]}=g \quad \text { et } \quad f_{i}(t)=\varphi_{i}(-t-1) \quad \text { pour } t \in[-2,-1]
$$

Considérons $\sigma: t \in[0,1] \mapsto(t-2, t-2) \in[-2,2]^{2}$. Alors

$$
\left(f_{1} \times f_{2}\right) \circ \sigma([0,1]) \supset K^{\prime} \times K^{\prime}
$$

de sorte que :

$$
h_{\text {top }}^{1}\left(f_{1} \times f_{2}\right)=h_{\text {top }}\left(f_{1}\right)+h_{\text {top }}\left(f_{2}\right)>h_{\text {top }}\left(f_{1}\right)=h_{\text {top }}\left(f_{2}\right) \text {. }
$$

En particulier, $h_{\text {top }}^{1}\left(f_{1} \times f_{2}\right)=h_{\text {top }}\left(f_{1} \times f_{2}\right)$.

Passons à la preuve du théorème 3.2. Il est évident que

$$
h_{\text {top }}^{k}\left(f_{1} \times \cdots \times f_{d}\right) \geq h_{\text {top }}\left(f_{1}\right)+\cdots+h_{\text {top }}\left(f_{k}\right) .
$$

Nous montrons l'inégalité réciproque. Soit $\alpha>0$ arbitrairement petit. Soit $\epsilon>0$ suffisamment petit par rapport à $\alpha$ (on verra combien petit ci-dessous). En premier lieu, on réduit le calcul de l'entropie d'une partie $S \subset[0,1]^{d}$ au calcul de ses intersections avec un réseau d'hyperplans. 
Lemme 3.4. - Pour $\epsilon>0$ assez petit, il existe des ensembles $E_{\text {in }} \subset[0,1]$ (avec $1 \leq i \leq d$ et $n \geq 1$ ) tels que:

(1) chaque cellule du réseau d'hyperplans $x_{i} \in E_{\text {in }}$, avec $1 \leq i \leq d$, i.e., chaque ensemble de la forme:

$$
\left\{\left(x_{1}, \ldots, x_{d}\right) ; \forall i=1, \ldots, d, u_{i} \leq x_{i} \leq v_{i}\right\}
$$

pour $u_{i}<v_{i}$ deux éléments consécutifs de $E_{\text {in }} \cup\{0,1\}$, est contenue dans une $\left(\frac{1}{2} \epsilon, n\right)$-boule définie par $f_{1} \times \ldots \times f_{d}$;

(2) $\# E_{\text {in }} \leq e^{\left(h_{\mathrm{top}}\left(f_{i}\right)+\alpha\right) n}$ pour $n$ grand (i.e., $\left.n \geq n_{0}(\alpha, \epsilon)\right)$.

Remarque 3.5. - C'est pour ce lemme qu'on a besoin de la régularité de $f$.

Preuve. - Ce lemme découle de l'existence, pour chaque entier $n$ assez grand, chaque $i=1, \ldots, d$, de $E_{i n}=\left\{x_{0}=0<x_{1}<\cdots<x_{N}=1\right\}$, de cardinalité $N$ bornée comme dans (2) ci-dessus et tel que :

$$
\forall k=0,1, \ldots, N-1, \quad\left[x_{k}, x_{k+1}\right] \subset B_{n}\left(x_{k}, \epsilon / 2 \sqrt{d}\right),
$$

la $n$-boule étant définie par rapport à $f_{i}$.

- Si $f_{i}$ est monotone par morceaux, l'existence d'un tel ensemble $E_{i n}$ est une conséquence immédiate des deux faits suivants :

(1) le nombre d'intervalles de monotonie de $f_{i}^{n}$ est borné, pour $n$ grand, par $\mathrm{e}^{\left(h_{\mathrm{top}}\left(f_{i}\right)+\frac{1}{2} \alpha\right) n}$;

(2) un intervalle de monotonie $I$ (i.e., de monotonie et de continuité) pour $f_{i}^{n}$ se décompose en au plus $3 \sqrt{d} \epsilon^{-1} n+1 \leq \mathrm{e}^{\left(\frac{1}{2} \alpha\right) n}$ sousintervalles, chacun contenu dans une $(\epsilon / 2 \sqrt{d}, n)$-boule.

L'assertion (1) est facile et bien connue [19]. On peut la redémontrer en considérant l'extension de $\left([0,1], f_{i}\right)$ définie par le doublement de toutes les extrémités des intervalles de monotonie et de leurs pré-images d'ordre quelconque (on munit l'ensemble obtenu de l'ordre naturel et de la topologie correspondante). C'est un compact métrique coïncidant avec $[0,1]$ à un ensemble dénombrable près. L'application $f_{i}$ s'étend naturellement à cet ensemble. D'après le principe variationnel, l'entropie topologique de ce système étendu est la même que celle de $f_{i}$, les deux systèmes ayant les mêmes mesures de probabilités invariantes et ergodiques non-atomiques. Maintenant (1) est simplement l'expression de l'entropie topologique en fonction du recouvrement ouvert du système étendu par les intervalles de monotonie.

$$
\text { TOME } 126-1998-\mathrm{N}^{\circ} 1
$$


Considérons (2). On peut définir les sous-intervalles de (2) comme les composantes connexes de

$$
I \backslash \bigcup_{0 \leq \ell<n} f_{i}^{-\ell}\left(\left\{\frac{\epsilon}{3 \sqrt{d}}, 2 \frac{\epsilon}{3 \sqrt{d}}, \ldots, m \frac{\epsilon}{3 \sqrt{d}}\right\}\right)
$$

avec $m$ la partie entière de $1 / \frac{\epsilon}{3 \sqrt{\mathrm{d}}}$.

- Si $f_{i}$ est une application $C^{\infty}$ quelconque, $\left(^{*}\right)$ découle du lemme 2.3 de [22] qui montre que, si $\epsilon>0$ est assez petit, on peut trouver, pour tout $x \in[0,1]$ et tout $n$ assez grand, une union $K$ de $\mathrm{e}^{\alpha n}$ intervalles telle que $B_{n}(x, \epsilon / 4 \sqrt{d}) \subset K \subset B_{n}(x, \epsilon / 2 \sqrt{d})$. En effet, ces intervalles sont, à un changement d'échelle près, les images des reparamétrisations $\tilde{\sigma} \circ \psi_{n j}$, $1 \leq j \leq \kappa^{n}$, de $\tilde{\sigma}=\operatorname{Id}_{[0,1]}$ dans les notations du lemme de Y. Yomdin. Comme d'habitude, pour obtenir la borne par $\mathrm{e}^{\alpha n}$, on doit considérer un ordre de différentiabilité et un ordre d'itération suffisament élevés.

Divisons $[0,1]^{d}$ par les hyperplans $x_{i} \in E_{i n}$. A cause de $\left(^{*}\right)$, chaque cellule est incluse dans une $\left(\frac{1}{2} \epsilon, n\right)$-boule. Donc, pour borner le nombre de $\left(\frac{1}{2} \epsilon, n\right)$-recouvrement $r\left(\frac{1}{2} \epsilon, n, S\right)$ d'un ensemble $S \subset[0,1]^{d}$ il suffit de compter le nombre de cellules qu'il rencontre. Le lemme est démontré.

Revenons à la preuve du théorème. Considérons un disque $C^{\infty}$

$$
\sigma:[-1,1]^{k} \longrightarrow[0,1]^{d}
$$

Soit $M$ une constante de Lipschitz commune aux $f_{i}$.

Fixons un entier $s \geq 1$ si grand que $\frac{k}{s} \log M<\alpha$. Soit

$$
K=\max _{u \in[-1,1]^{k}}\left\|D^{s} \sigma(u)\right\|
$$

$D^{s} \sigma$ étant la différentielle d'ordre $s$ et la norme étant la norme habituelle pour les opérateurs multi-linéaires par rapport à la structure euclidienne canonique de $\mathbb{R}^{k}$ et $\mathbb{R}^{d}$. Divisons $[-1,1]^{k}$ en cellules de côté au plus $\left(\epsilon K^{-1} d^{-1 / 2}\right)^{1 / s} \times M^{-n / s}$. Il y a au plus

$$
\left(d^{1 / 2 s} K^{1 / s} \epsilon^{-1 / s}+1\right)^{k} M^{\frac{k}{s} n} \leq \mathrm{C}^{\mathrm{te}} \cdot \mathrm{e}^{\alpha n}
$$

cellules comme ceci (avec $\mathrm{C}^{\text {te }}$ dépendant seulement de $\alpha, \epsilon, M, K, d$ ). Donc il suffira de borner le nombre de recouvrement de l'image par $\sigma$ d'une telle cellule $Q$. 
Soit $P_{\sigma}$ le polynôme défini par l'expansion de Taylor de degré $s-1$ à partir de $u_{*}$, le centre de la cellule. Clairement, pour tous les points $u$ de la cellule :

$$
\left\|\sigma(u)-P_{\sigma}(u)\right\| \leq K\left\|u-u_{*}\right\|^{s} \leq \frac{1}{2} \epsilon M^{-n} .
$$

Ceci implique que $\sigma(u)$ appartient à $B_{n}\left(P_{\sigma}(u), \frac{1}{2} \epsilon\right)$. Il suffit donc de borner le nombre de recouvrement $r\left(\frac{1}{2} \epsilon, n, P_{\sigma}(Q)\right)$ et donc, par le lemme ci-dessus, il suffit de compter le nombre de cellules du réseau $x_{i} \in E_{\text {in }}$ qui sont rencontrées par $P_{\sigma}(Q)$. Quitte à déplacer $P_{\sigma}(Q)$ d'une distance arbitrairement petite, on peut s'arranger pour que toutes les cellules rencontrées le soient dans leur intérieur aussi. Le nombre de recouvrement est donc borné par le nombre de composante connexe de

$$
P_{\sigma}(Q) \backslash\left\{x \in[0,1]^{d} ; x_{i} \in E_{i n}(i=1, \ldots, d)\right\} .
$$

On a réduit notre problème au lemme suivant de géométrie semialgébrique. Rappelons qu'un ensemble semi-algébrique est une partie d'un $\mathbb{R}^{d}$, avec $d \geq 1$, définie par un nombre fini d'équations et d'inéquations polynômiales. Le degré d'un ensemble d'équations et d'inéquations est la somme des degrés totaux des polynômes en question. Le degré d'un ensemble semi-algébrique est le plus petit degré des ensembles d'équations et d'inéquations le définissant. La dimension d'un ensemble semi-algébrique est le plus grand entier $p$ tel que l'ensemble contienne une partie homéomorphe à $\mathbb{R}^{p}$.

Lemme 3.6. - Soit $A$ une partie semi-algébrique de $\mathbb{R}^{d}$ et soient $\mathcal{E}_{1}, \ldots, \mathcal{E}_{r}$ des collections de parties compactes et semi-algébriques de $\mathbb{R}^{d}$ avec $\# \mathcal{E}_{1} \geq \cdots \geq \# \mathcal{E}_{r}$. Supposons, pour chaque $i$, que les ensembles dans $\mathcal{E}_{i}$ sont deux à deux disjoints. Alors, en posant $t=\min (\operatorname{dim} A, r)$, le nombre de composantes connexes de $A \backslash \mathbb{E}$ ( $\mathbb{E}$ étant l'union des ensembles dans tous les $\mathcal{E}_{i}$ pour $\left.i=1, \ldots, r\right)$, est :

$$
N(A \backslash \mathbb{E}) \leq C \# \mathcal{E}_{1} \times \cdots \times \# \mathcal{E}_{t}
$$

avec $C$ un nombre dépendant seulement de $d, r$ et du plus grand degré des ensembles semi-algébriques $A, E \in \mathcal{E}_{i}$ pour $i=1, \ldots, d$.

Avant de prouver ce lemme, on en déduit le théorème.

Le théorème de Tarski-Seidelberg [7] implique que $A=P_{\sigma}(Q)$, comme image d'un semi-algébrique par une application polynômiale, est encore un semi-algébrique. De plus, le degré de cet ensemble est borné par un nombre dépendant seulement des dimensions des espaces et des degrés de l'ensemble initial et des polynômes qui définissent l'application polynômiale.

TOME $126-1998-\mathrm{N}^{\circ} 1$ 
Pour $i=1, \ldots, d$, soit $\mathcal{E}_{i}$ l'ensemble des hyperplans d'équation $x_{i}=\mathrm{C}^{\text {te }}$ avec $\mathrm{C}^{\text {te }} \in E_{i n}$. On peut supposer que $\# \mathcal{E}_{1} \geq \cdots \geq \# \mathcal{E}_{r}$.

Le lemme 3.6 donne :

$$
N\left(P_{\sigma}(Q) \backslash \mathbb{E}\right) \leq C \exp \left[\sum_{i=1}^{k}\left(h_{\mathrm{top}}\left(f_{i}\right)+\alpha\right) n\right],
$$

$C$ étant un nombre indépendant de $n$ et de $\sigma$. On en conlut :

$$
\begin{aligned}
r\left(\epsilon, n, \sigma\left([-1,1]^{k}\right)\right) & \leq \mathrm{e}^{\alpha n} \max _{Q} r(\epsilon, n, \sigma(Q)) \\
& \leq \mathrm{e}^{\alpha n} \max _{Q} r\left(\frac{1}{2} \epsilon, n, P_{\sigma}(Q)\right) \\
& \leq \mathrm{e}^{\alpha n} \max _{Q} N\left(P_{\sigma}(Q) \backslash \mathbb{E}\right) \\
& \leq \mathrm{C}^{\mathrm{te}} \cdot \mathrm{e}^{(k+1) \alpha n} \exp \left(\sum_{i=1}^{k} h_{\mathrm{top}}\left(f_{i}\right)\right) n
\end{aligned}
$$

où le nombre $\mathrm{C}^{\text {te }}$ est indépendant de $n$ et ne dépend de $\sigma$ qu'au travers de $K=\max \left\|d^{s} \sigma(u)\right\|$.

Ceci donne la borne annoncée sur $h_{\text {top }}\left(\sigma, f_{1} \times \ldots \times f_{d}\right)$ pour tous les disques réguliers $\sigma$. On a donc majoré $h_{\text {top }}^{k}\left(f_{1} \times \ldots \times f_{d}\right)$, ce qui termine la preuve du théorème 3.2 .

Remarque 3.7. - La borne sur $r(\epsilon, n, \operatorname{Im}(\sigma))$ obtenue ci-dessus est uniforme pour $\sigma$ de taille $C^{s}$ bornée (pour $s$ grand, dépendant de $F_{0}$, de $d$ et $\operatorname{de} \alpha$ ).

On renvoie à [7] pour une présentation de la géométrie semi-algébrique réelle. Nous utiliserons seulement les deux faits basiques suivants.

Si $A \subset \mathbb{R}^{d}$ est un semi-algébrique, alors :

(SA1) $A$ est l'union d'un nombre borné de sous-variétés connexes qui sont elles-mêmes semi-algébriques de degré borné. De plus, chacune de ces sous-variétés est disjointe de la fermeture des autres. Nous appelons ces sous-variétés les pièces de $A$.

(SA2) La frontière (topologique) $\partial_{B} A$ de $A$ comme partie d'un autre semi-algébrique $B$ est elle-même semi-algébrique de degré borné et de dimension au plus $\min (\operatorname{dim} A, \operatorname{dim} B-1)$.

Ici borné veut dire borné par un nombre dépendant seulement des degrés de $A$ et de $B$ et de la dimension $d$. Les sous-variétés considérées sont sans bords.

On utilisera le fait facile suivant : 
Lemme 3.8. - Soient $\Sigma \subset M \subset \mathbb{R}^{d}$ deux sous-variétés connexes et soit $K \subset \mathbb{R}^{d}$ un compact. Supposons que $K$ ne rencontre pas $\Sigma$. Alors au plus deux composantes connexes $U$ de $M \backslash(K \cup \Sigma)$ peuvent toucher $\Sigma$, i.e., vérifier : $\bar{U} \cap \Sigma \neq \emptyset$.

Preuve. - Remarquons d'abord que si $\operatorname{dim}(\Sigma)=\operatorname{dim}(M)$ alors $\Sigma$ est ouvert dans $M$; donc la fermeture de $M \backslash(K \cup \Sigma)$ est disjointe de $\Sigma$ : le lemme est trivial dans ce cas.

Supposons maintenant $\operatorname{dim}(\Sigma)<\operatorname{dim}(M)$. Procédons par l'absurde : supposons que trois composantes distinctes $C_{1}, C_{2}, C_{3}$ de l'ensemble $M \backslash(K \cup \Sigma)$ touchent $\Sigma$. Soit $x_{i}$ un point d'intersection de $\overline{C_{i}}$ avec $\Sigma$ pour $i=1,2,3$. Pour $r>0$ suffisamment petit, $\left(B\left(x_{3}, r\right) \cap M\right) \backslash \Sigma$ est difféomorphe à

$$
\mathbb{R}^{\operatorname{dim} M} \backslash\left\{t \in \mathbb{R}^{\operatorname{dim} M} ; t_{\operatorname{dim} \Sigma+1}=\cdots=t_{\operatorname{dim} M}=0\right\} .
$$

En particulier, $\left(B\left(x_{3}, r\right) \cap M\right) \backslash \Sigma$ a au plus deux composantes connexes. De plus, comme $r$ est petit, $B\left(x_{3}, r\right)$ ne rencontre pas $K$. Donc chaque composante de $\left(B\left(x_{3}, r\right) \cap M\right) \backslash \Sigma$ est incluse dans une composante de $M \backslash(\Sigma \cup K)$.

Soit $i=1$ ou 2. Soit $\gamma_{i}$ un chemin dans $\Sigma$ joignant $x_{i}$ à $x_{3}$. Comme $\Sigma$ est de codimension au moins 1 dans $M$, on peut pousser $\gamma_{i}$ de sorte que $\gamma_{i}$ n'intersecte $\Sigma$ qu'en $x_{i}$ et en $x_{3}$ sans rencontrer $K$ (qui est à une distance positive de $\gamma_{i} \subset \Sigma$ ). Ainsi $\gamma_{i}$ est contenu dans $C_{i}$ (à l'exception de ses extrémités $x_{i}$ et $x_{3}$ ).

Le point $x_{3}$ appartient donc à la frontière des trois composantes $C_{1}, C_{2}, C_{3}$ qui doivent toutes les trois rencontrer $B\left(x_{3}, r\right)$. Mais on a vu que $B\left(x_{3}, r\right) \cap(M \backslash(\Sigma \cup K))$ a au plus deux composantes. C'est la contradiction.

Preuve du lemme 3.8. - En utilisant (SA1), on voit qu'on peut supposer que $A$ est une sous-variété connexe de dimension $p$.

Si $r=0$ ou $p=0$, alors $N(A \backslash \mathbb{E}) \leq 1$, et la majoration annoncée est évidente. Soient $p, r \geq 1$. Supposons la propriété pour tous $p^{\prime}, r^{\prime} \geq 0$ vérifiant $p^{\prime}+r^{\prime}<p+r$. Soit $\mathbb{F}$ l'union des ensembles dans $\mathcal{E}_{1}, \ldots, \mathcal{E}_{r-1}$. Par l'hypothèse de récurrence on a, avec $s=\min (p, r-1)$ :

$$
N(A \backslash \mathbb{F}) \leq C \# \mathcal{E}_{1} \cdots \# \mathcal{E}_{s} .
$$

L'augmentation $N(A \backslash \mathbb{E})-N(A \backslash \mathbb{F})$ est bornée par le nombre de nouvelles composantes, i.e., de composantes de $A \backslash \mathbb{E}$ qui ne sont pas des composantes de $A \backslash \mathbb{F}$. Fixons une nouvelle composante $U$; celle-ci vérifie $\partial_{A \backslash \mathbb{F}} U \neq \emptyset$. Donc, pour un $E_{*} \in \mathcal{E}_{r}, \bar{U}$ doit rencontrer $\partial_{A} E_{*} \backslash \mathbb{F} \supset \partial_{A \backslash \mathbb{F}} E_{*}$.

TOME $126-1998-\mathrm{N}^{\circ} 1$ 
Décomposons $\partial_{A} E_{*}$ en pièces d'après (SA1) :

- soit $D_{0}$ une pièce de $\partial_{A} E_{*}$ telle que $\bar{U} \cap D_{0} \backslash \mathbb{F} \neq \emptyset$;

- soit $D_{1}$ une composante de $D_{0} \backslash \mathbb{F}$ touchée par $U$;

- soit $D_{2}=\overline{\partial_{A} E_{*} \backslash D_{1}}$.

Vérifions que $D_{1} \cap D_{2}=\emptyset$. En effet, $D_{2}=\overline{\partial_{A} E_{*} \backslash D_{0}} \cup \overline{D_{0} \backslash D_{1}}$. Le premier terme de cette union est, par définition d'une pièce, disjoint de $D_{0}$, donc de $D_{1}$. Quant au second terme, remarquons que $D_{0} \backslash D_{1}$ est fermé dans $D_{0}$, donc la fermeture n'ajoute pas de points de $D_{0}$, donc de $D_{1}$. Ainsi $D_{1} \cap D_{2}=\emptyset$.

Vérifions que $D_{1} \cup D_{2}=\partial_{A} E_{*}$ modulo le complémentaire de $A$. En effet, $D_{1} \cup D_{2} \supset \partial_{A} E_{*}$. Réciproquement, on a $D_{1} \subset D_{0} \subset \partial_{A} E_{*}$ et $D_{2}=\overline{\partial_{A} E_{*} \backslash D_{1}} \subset \overline{\partial_{A} E_{*}}$. Mais $\partial_{A} E_{*}$ est fermé dans $A$, la fermeture n'ajoute donc pas de points de $A$. Ceci démontre l'affirmation.

Posons :

$$
K=\mathbb{F} \cup \bigcup_{\substack{E \in \mathcal{E}_{r} \\ E \neq E_{*}}} E \cup D_{2}
$$

On a :

$$
A \backslash\left(K \cup D_{1}\right)=A \backslash\left(\mathbb{F} \cup \bigcup_{\substack{E \in \mathcal{E}_{r} \\ E \neq E_{*}}} E \cup \partial_{A} E_{*}\right) .
$$

Ce dernier ensemble a au moins autant de composantes que $A \backslash \mathbb{E}$.

En appliquant le lemme précédent à $\Sigma=D_{1}, M=A$ et l'ensemble $K$ défini ci-dessus, on trouve qu'au plus deux composantes de $A \backslash \mathbb{E}$ peuvent rencontrer $D_{1}$. Il suffit donc de majorer le nombre de possibilités pour $D_{1}$.

D'après (SA1), pour $E_{*} \in \mathcal{E}_{r}$ fixé, le nombre de $D_{0}$ est majoré par un nombre borné (i.e., dépendant seulement des degrés de $A$ et de $E_{*}$ ). Le nombre de $D_{1}$ est donné par $N\left(D_{0} \backslash \mathbb{F}\right)$. Comme $\mathbb{F}$ est constitué de $(r-1)$ familles et que $\operatorname{dim} D_{0}<\operatorname{dim} A$, on peut appliquer l'hypothèse de récurrence et, en remarquant que les $D_{0}$ sont de degré borné, on trouve :

$$
N\left(D_{0} \backslash \mathbb{F}\right) \leq C^{\prime} \# \mathcal{E}_{1} \cdots \# \mathcal{E}_{q}
$$

avec $q=\min \left(\operatorname{dim}\left(D_{0}\right), r-1\right) \leq \min (p-1, r-1)$ et $C^{\prime}$ un nombre borné.

Le nombre de $D_{1}$ quand $E_{*}$ parcourt $\mathcal{E}_{r}$ est borné par \# $\mathcal{E}_{r}$ fois la majoration précédente, donc par :

$$
C^{\prime \prime} \# \mathcal{E}_{1} \cdots \# \mathcal{E}_{t}
$$

avec $C^{\prime \prime}$ un nombre borné, vu que $t=\min (p, r) \geq q+1$. En multipliant ceci par 2, on obtient un majorant du nombre de nouvelles composantes. En sommant avec $\left(^{*}\right)$ (remarquons que $t \geq s$ ) on finit la preuve. 


\subsection{Propriétés de semi-continuité.}

On veut étendre les estimations précédentes aux petites perturbations.

On montre d'abord que $F_{0}=f_{1} \times \cdots \times f_{d}$ est un point de semi-continuité inférieure de l'entropie topologique $F \mapsto h_{\text {top }}(F)$ dans la topologie $C^{0}$.

Remarque. - L'application $F \mapsto h_{\text {top }}(F)$ n'est pas semi-continue inférieurement en tout $F$. Par exemple, considérons les

$$
F_{\lambda}:(x, y) \longmapsto(\lambda x, 4 x y(1-y))
$$

sur le carré $[0,1]^{2}$ pour $\lambda \in[0,1]$. Pour $\lambda=1$, l'entropie topologique est $\log 2$, alors que pour tout $\lambda<1$, elle est nulle.

En $F=F_{0}$, la semi-continuité inférieure découle du résultat unidimensionnel suivant :

ThÉorème 3.9 (M. Misiurewicz [18]). — Soit $f:[0,1] \rightarrow[0,1]$ continue. Pour tout $\epsilon>0$, il existe $N$ intervalles $J_{1}, \ldots, J_{N}$ fermés, disjoints, et un entier $K$ tels que:

(1) pour chaque $i$, l'intérieur de $f^{K}\left(J_{i}\right)$ contient $J_{1} \cup \ldots \cup J_{N}$;

(2) $\log N / K>h_{\text {top }}(f)-\epsilon$.

En effet, ceci implique que $F_{0}$ contient un sous-système isomorphe à un sous-shift de type fini d'entropie supérieure à $h_{\text {top }}\left(F_{0}\right)-\epsilon$ et que ce sous-système est stable vis-à-vis des perturbations $C^{0}$. Donc $h_{\text {top }}(F) \geq h_{\text {top }}\left(F_{0}\right)-\epsilon$ pour $F$ proche de $F_{0}$, ce qui prouve la semicontinuité inférieure.

On considère maintenant la semi-continuité supérieure de $F \mapsto h_{\text {top }}^{d-1}(F)$.

Rappelons la définition de l'entropie topologique de dimension $k$ :

$$
h_{\mathrm{top}}^{k}(F)=\sup _{\sigma} \lim _{\epsilon \searrow 0} \limsup _{n \rightarrow \infty} \frac{1}{n} \log r(\epsilon, n, \operatorname{Im}(\sigma)),
$$

la borne supérieure étant prise sur les applications $\sigma:[-1,1]^{k} \rightarrow[0,1]^{d}$ de classe $C^{\infty}$.

On définit $H_{\text {top }}^{k}(F)$, une version de $h_{\text {top }}^{k}(F)$ uniforme par rapport aux $\sigma$ de taille $C^{s}$ fixée $\|\sigma\|_{s}=\max _{1 \leq t \leq s}\left\|D^{t} \sigma\right\|$ :

$$
H_{\mathrm{top}}^{k}(F)=\lim _{s \rightarrow \infty} \lim _{\epsilon \searrow 0} \limsup _{n \rightarrow \infty} \frac{1}{n} \log r_{s}^{k}(\epsilon, n)
$$

avec

$$
r_{s}^{k}(\epsilon, n)=\sup _{\substack{\sigma:[-1,1]^{k} \rightarrow[0,1]^{d} \\\|\sigma\|_{s} \leq 1}} r(\epsilon, n, \operatorname{Im}(\sigma))
$$

TOME $126-1998-\mathrm{N}^{\circ} 1$ 
En utilisant les techniques de Y. Yomdin [22] pour les nombres de recouvrements au lieu des volumes, on a montré la propriété de «quasi sousmultiplicativité» suivante :

Proposition 3.10 (voir [2, p. 80]). - Soit $f: V \rightarrow V$ une application $C^{s}$ sur une variété compacte munie d'une structure riemmannienne quelconque. Pour tout $\alpha>0$, tout $0<\epsilon<\epsilon_{0}$ et tous $n$ et $m \geq 0$, on a

$$
r_{s}^{k}(\epsilon, n m) \leq \frac{C}{\epsilon^{k}}\left(M(f)^{\frac{k}{s}} e^{\alpha}\right)^{n m} r_{s}^{k}\left(\epsilon_{0}, n\right)^{m}
$$

où :

(1) $M(f) \stackrel{\text { def }}{=} \sup _{x} \max \left(\left\|f^{\prime}(x)\right\|, 1\right)$;

(2) $C<\infty$ ne dépend que de $V, s, \alpha$;

(3) $\epsilon_{0}>0$ ne dépend que de $V$ et de la norme des $s$ premières dérivées de $f$.

De plus, $\epsilon_{0}$ et $C$ peuvent être choisis continuement en fonction de $f$.

Ceci implique immédiatement le résultat suivant (énoncé avec une preuve détaillée dans $[2,7.14]$ :

Corollaire 3.11 (voir M. Gromov [9, remarque à la fin de 3.9]). L'application $F \mapsto H_{\mathrm{top}}^{k}(F)$ est semi-continue supérieurement dans la topologie $C^{\infty}$.

Nous affirmons que $H_{\text {top }}^{k}\left(F_{0}\right)=h_{\text {top }}^{k}\left(F_{0}\right)$. Pour tout $F$, on a clairement $H_{\text {top }}^{k}(F) \geq h_{\text {top }}^{k}(F)$. Pour $F=F_{0}$, l'inégalité réciproque résulte de la remarque 3.7 figurant à la fin de la preuve du théorème 3.2 : l'estimation de ce théorème s'applique en fait à $H_{\text {top }}^{k}\left(F_{0}\right)$.

Remarque 3.12. - En fait, on peut montrer que pour toute application $F$ de classe $C^{\infty}$ sur une variété compacte, les deux entropies coïncident toujours : $h_{\mathrm{top}}^{k}(F)=H_{\mathrm{top}}^{k}(F)$.

Maintenant, étant donné $\epsilon>0$, pour $F$ assez proche de $F_{0}$ dans la topologie $C^{\infty}$, on obtient :

$$
h_{\mathrm{top}}^{k}(F) \leq H_{\mathrm{top}}^{k}(F) \leq H_{\mathrm{top}}^{k}\left(F_{0}\right)+\epsilon=h_{\mathrm{top}}^{k}\left(F_{0}\right)+\epsilon .
$$

\subsection{Conclusion.}

Grâce au théorème 3.2 , on voit que

$$
h_{\mathrm{top}}\left(F_{0}\right)=h_{\mathrm{top}}^{d-1}\left(F_{0}\right)+\min _{i} h_{\mathrm{top}}\left(f_{i}\right) .
$$

BULLETIN DE LA SOCIÉTÉ MATHÉMATIQUE DE FRANCE 
Donc, pour tout $\epsilon>0$, on a

$$
h_{\mathrm{top}}\left(F_{0}\right)>h_{\mathrm{top}}^{d-1}\left(F_{0}\right)+\min _{i} h_{\mathrm{top}}\left(f_{i}\right)-\epsilon .
$$

Les propriétés de semi-continuité de la section précédente montrent que cette inégalité reste vraie dans un voisinage $C^{\infty}$ de $F_{0}$. C'est le contenu du théorème 3.1 .

\section{Partition naturelle}

\subsection{Construction.}

Soit $F:[0,1]^{d} \rightarrow[0,1]^{d}$ une application comme dans le théorème principal : une petite perturbation fibrée du produit direct $F_{0}=f_{1} \times \cdots \times f_{d}$, les orbites critiques des $f_{i}$ étant non-dégénérées (1.1).

On suppose que les applications $f_{i}$ ont été normalisées :

$$
f_{i}^{-1}(\{0,1\}) \subset\{0,1\} \quad \text { et } \quad f_{i}^{\prime}(0), f_{i}^{\prime}(1) \neq 0 .
$$

On peut toujours le faire sans modifier l'ensemble des mesures de probabilités invariantes et ergodiques, à l'exception de l'addition éventuelle de mesures atomiques qui ne nous concernent pas.

On considère l'ensemble critique :

$$
C(F) \stackrel{\text { def }}{=}\left\{p \in[0,1]^{d} ; \operatorname{det}\left(F^{\prime}(p)\right)=0\right\} .
$$

La partition naturelle de $F$ est définie comme l'ensemble $P$ des composantes connexes de $[0,1]^{d} \backslash C(F)$.

Comme $F$ est fibrée, son jacobien est de la forme :

$$
\frac{d \bar{f}_{1}}{d x_{1}}\left(x_{1}\right) \frac{\partial \bar{f}_{2}}{\partial x_{2}}\left(x_{1}, x_{2}\right) \cdots \frac{\partial \bar{f}_{d}}{\partial x_{d}}\left(x_{1}, \ldots, x_{d}\right) .
$$

Chacune de ces dérivées est donc non-nulle et de signe fixé sur chaque élément de la partition $P$. Par conséquent, pour chaque $A \in P$,

$$
F: \bar{A} \longrightarrow \overline{F(A)}
$$

est un homéomorphisme. En particulier $\left([0,1]^{d}, P, F\right)$ est un système dynamique inversible par morceaux.

Remarquons que $C\left(F_{0}^{k}\right)$, pour chaque $k \geq 1$, est l'union d'un nombre fini d'hyperplans parallèles aux axes de coordonnées. La condition de non

TOME $126-1998-\mathrm{N}^{\circ} 1$ 
dégénérescence (1.1) avec la normalisation garantit que $C\left(F^{k}\right)$ dépend continûment de $F$ sur un petit voisinage de $F_{0}$ (dépendant de $k$ ). Plus précisément, il existe un nombre fini d'applications $C^{\infty}$,

$$
\sigma_{F}:[0,1]^{d-1} \rightarrow[0,1]^{d}
$$

dépendant continûment de $F$, telles que $C\left(F^{k}\right)$ est l'union de leurs images et les applications $\sigma_{F_{0}}$ sont affines et correspondent aux hyperplans constituant $C\left(F_{0}^{k}\right)$.

Cette situation a les conséquences triviales mais importantes suivantes, pour toute application $F$ fibrée et suffisamment proche de $F_{0}$.

- La première est que l'intersection de tout $A \in P$ avec tout intervalle vertical $\{x\} \times[0,1]$ (où $x \in[0,1]^{d-1}$ ) est un intervalle vertical. En utilisant la monotonie de $f$ restreinte à une telle intersection, on voit que cette propriété est vraie pour $A \in P^{n}$ pour tout $n \geq 1$.

- La seconde est que, avec un abus de notation évident :

$$
\operatorname{mult}\left([0,1]^{d} \backslash C\left(F^{k}\right)\right)=2^{d} .
$$

Or la partition naturelle de $F^{k}$ est plus fine que la $k$-ième itération $P^{k}$ de la partition $P$. Donc ( $F$ étant supposé proche de $F_{0}$ ), on a :

$$
h_{\text {mult }}(P, F) \leq \frac{1}{k} \log \operatorname{mult}\left(P^{k}\right) \leq \frac{d \cdot \log 2}{k} .
$$

Pour $k$ assez grand, $h_{\text {mult }}(P, F)$ est donc majoré par $\frac{1}{3} \min _{i} h_{\text {top }}\left(f_{i}\right)$. Rappelons (théorème 3.1 ) que $h_{\mathrm{top}}(F)>h_{\mathrm{top}}^{d-1}(F)+\frac{1}{3} \min _{i} h_{\mathrm{top}}\left(f_{i}\right)$. On a donc l'inégalité entropique (2.3).

On a montré :

Lemme 4.1. - Il existe un voisinage $\mathcal{U}$ de $f_{1} \times \ldots \times f_{d}$ dans la topologie $C^{\infty}$ tel que toute application $F \in \mathcal{U}$ admet une partition naturelle $P$ telle que :

(1) $\left([0,1]^{d}, P, F\right)$ est inversible par morceaux;

(2) la frontière de la partition $\partial P$ est une union finie d'hypersurfaces aussi régulières que $F$;

(3) on a l'inégalité entropique :

$$
h_{\text {top }}(\partial P, f)+h_{\text {mult }}(P, f)<h_{\text {top }}(f) \text {; }
$$

(4) l'intersection de tout $A \in P^{n}$ avec un intervalle vertical est un intervalle vertical. 


\section{2. $P$ sépare presque toutes les orbites.}

On montre la :

Proposition 4.2. - Soit F comme dans le théorème principal. Alors, pour h-presque tout $x \in[0,1]^{d}$, on $a$ :

$$
\lim _{n \rightarrow \infty} \operatorname{diam}\left(P^{n}(x)\right)=0 .
$$

Remarque 4.3. - La formule (*) peut tomber en défaut sur un ensemble assez grand. On peut construire des applications de l'intervalle, $f$ et $g$, satisfaisant les hypothèses du théorème principal telles qu'il existe des perturbations fibrées arbitrairement petites $F$ de $f \times g$ qui admettent des mesures de probabilités invariantes et ergodiques de fibres au-dessus de $f$ non-atomiques, mesures par rapport auxquelles $P$ ne sépare pas presque toutes les orbites. Mais ces mesures ont petite entropie, en fait :

$$
h_{\mu}(F)=h_{\pi_{1} \mu}(f) \leq h_{\mathrm{top}}(f) .
$$

On procède par récurrence sur la dimension $d$ du produit.

- Si $d=1$ le résultat est bien connu : la partition naturelle définie ci-dessus est une partition en intervalles de monotonie, elle sépare donc presque toutes les orbites (excepté peut-être par rapport à des mesures concentrées sur des orbites périodiques, donc d'entropie nulle).

- Soit $d \geq 2$. Supposons le résultat pour $G:[0,1]^{d-1} \rightarrow[0,1]^{d-1}$ définie par

$$
G\left(x_{1}, \ldots, x_{d-1}\right)=\pi\left(F\left(x_{1}, \ldots, x_{d-1}, 0\right)\right)
$$

où

$$
\pi:\left(x_{1}, \ldots, x_{d}\right) \longmapsto\left(x_{1}, \ldots, x_{d-1}\right) .
$$

Pour pouvoir exploiter l'hypothèse de récurrence, nous avons besoin de savoir qu'une mesure de grande entropie pour $F$ se projette par $\pi$ sur une mesure de grande entropie pour $G$. Cela découle du lemme suivant, qui étend la semi-continuité supérieure de l'entropie topologique du cas d'une application fixée (le cas classique dû à S. Newhouse [20]) au cas d'une composition aléatoire :

Lemme 4.4. - Soit $f: M \rightarrow M$ une application $C^{\infty}$ d'une variété compacte. Pour tout $\alpha>0$, il existe un voisinage $\mathcal{U}$ de $f$ dans la topologie $C^{\infty}$ tel que la propriété suivante a lieu.

Soit $G: X \rightarrow X$ une application continue d'un compact métrique $X$. Soit $F: X \times M \rightarrow X \times M$ une extension de $G$ par des applications proches

TOME $126-1998-\mathrm{N}^{\circ} 1$ 
de $f$, i.e., $F(x, y)=\left(G(x), f_{x}(y)\right)$ avec $f_{x} \in \mathcal{U}$ dépendant continûment de $x$. Soit $\mu$ une mesure de probabilité $F$-invariante se projetant sur $\nu$ $G$-invariante. On $a$ :

$$
h_{\mu}(F) \leq h_{\nu}(G)+h_{\text {top }}(f)+\alpha .
$$

Remarque 4.5. - Si $X$ est réduit à un point, c'est le résultat de Newhouse. En particulier, il est bien connu qu'on ne peut pas remplacer la topologie $C^{\infty}$ par la topologie $C^{r}$ si $r<\infty$.

Preuve du lemme. - On va majorer l'entropie de $\mu$ en utilisant la formule de l'entropie de Katok [14], c'est-à-dire, en trouvant un $(\epsilon, n N)$ recouvrement de $(X \times M, F, \mu)$, i.e., une collection de $(\epsilon, n N)$-boules dont l'union est de $\mu$-mesure supérieure à une constante indépendante de $n$ et ceci pour $\epsilon>0$ quelconque et tout $n \geq 0$. L'entier $N$ sera défini au cours de la preuve.

Toute suite d'applications de $M, f_{1}, f_{2}, \ldots, f_{n-1}$, définit des orbites : les suites $x, f_{1}(x), f_{2} \circ f_{1}(x), \ldots, f_{n-1} \circ \cdots \circ f_{1}(x)$ pour $x \in M$. On définit de manière évidente à partir de là les $(\epsilon, n)$-boules et les $(\epsilon, n)$ recouvrements pour une telle suite d'applications. En utilisant les techniques de Y. Yomdin, nous avons montré [2, 7.17] (voir aussi [3, preuve du th. 2.2]) que, pour tout $\alpha>0$, il existe un voisinage $\mathcal{U}_{0}$ de $f$ dans la topologie $C^{\infty}$ et $\epsilon_{0}>0$ tels que, pour tout $\epsilon>0$, le nombre de $(\epsilon, n)$-boules nécessaires pour recouvrir une $\left(\epsilon_{0}, n\right)$-boule quelconque $B_{n}\left(x, \epsilon_{0}\right)$ vérifie :

$$
r\left(\epsilon, n, B_{n}\left(\epsilon_{0}, n\right)\right) \leq C_{0}(\epsilon) \mathrm{e}^{\alpha n / 10}
$$

pour tout $n \geq 0$ et toute suite d'applications dans $\mathcal{U}_{0}$. Ici $C_{0}(\epsilon)<\infty$ est un nombre indépendant de $n$ et du choix des applications dans $\mathcal{U}_{0}$.

On commence par fixer $N_{0}$ si grand que $(M, f)$ admet un $\left(\frac{1}{3} \epsilon_{0}, N_{0}\right)$-recouvrement $\mathcal{S}_{*}^{N_{0}}$ par au plus $e^{\left(h_{\mathrm{top}}(f)+\alpha / 10\right) N_{0}}$ points.

On fixe maintenant $\mathcal{U} \subset \mathcal{U}_{0}$, un voisinage assez petit de $f$ pour que $\mathcal{S}_{*}^{N_{0}}$ soit un $\left(\frac{1}{2} \epsilon_{0}, N_{0}\right)$-recouvrement pour toute suite de $N_{0}$ applications $f_{i} \in \mathcal{U}$.

On fixe $\epsilon>0$. Prenons $N$ un multiple de $N_{0}$ si grand pour que l'on ait $C_{0}(\epsilon / 10) \leq e^{\alpha N / 10}$.

Par la continuité uniforme de $x \mapsto f_{x}$, il existe $0<\delta<\frac{1}{2} \epsilon$ tel que pour tous $x, x^{\prime} \in X$ :

$$
d_{N}\left(x, x^{\prime}\right)<\delta \Longrightarrow \forall y \in M, \forall k=0, \ldots, N-1, d\left(f_{x}^{k}(y), f_{x^{\prime}}^{k}(y)\right)<\frac{1}{10} \epsilon
$$


où

$$
\begin{gathered}
f_{x}^{k} \stackrel{\text { def }}{=} f_{G^{k-1} x} \circ \cdots \circ f_{x}, \\
d_{N}\left(x, x^{\prime}\right) \stackrel{\text { def }}{=} \max _{0 \leq n<N} d\left(G^{n} x, G^{n} x^{\prime}\right) .
\end{gathered}
$$

Pour chaque $x \in X$, choisissons un $\left(\frac{1}{10} \epsilon, N\right)$-recouvrement $\mathcal{S}_{x}^{N}$ de $M$ par rapport à la suite d'applications $f_{x}, f_{G x}, \ldots, f_{G^{N-1} x}$.

Maintenant, $\left(\# \mathcal{S}_{*}^{N_{0}}\right)^{N / N_{0}}$ majore la cardinalité d'un $\left(\epsilon_{0}, N\right)$-recouvrement minimal de $M$ par rapport à toute suite d'applications dans $\mathcal{U}$, donc par rapport à $f_{x}, \ldots, f_{G^{N-1} x}$ (on peut appliquer une variante du raisonnement sur $\# \mathcal{Q}$ du paragraphe suivant). Donc, en utilisant la majoration (4.6), on obtient :

$$
\# \mathcal{S}_{x}^{N} \leq C_{0}\left(\frac{1}{10} \epsilon\right) \mathrm{e}^{\frac{1}{10} \alpha N}\left(\# \mathcal{S}_{*}^{N_{0}}\right)^{N / N_{0}} \leq \mathrm{e}^{\left(h_{\mathrm{top}}(f)+\frac{3}{10} \alpha\right) N}
$$

Pour $m$ grand, prenons un $(\delta, m N)$-recouvrement $\mathcal{R}^{m N}$ de $(X, G, \nu)$ par au plus $e^{\left(h_{\nu}(G)+\frac{1}{10} \alpha\right) n}$ points. Posons :

$$
\mathcal{T}^{m N}=\left\{\left(x, y_{0}, \ldots, y_{m-1}\right) ; x \in \mathcal{R}^{m N}, y_{k} \in \mathcal{S}_{G^{k N} x}^{N}\right\}
$$

Clairement,

$$
\# \mathcal{T}^{m N} \leq e^{\left(h_{\nu}(G)+h_{\mathrm{top}}(f)+\frac{4}{10} \alpha\right) m N} .
$$

Soit $\mathcal{Q}$ un ensemble $(\epsilon, m N)$-séparé maximal dans $\bigcup_{x \in \mathcal{R}^{m N}} B_{m N}(x, \epsilon) \times M$. La maximalité implique que $\mathcal{Q}$ est aussi un $(\epsilon, m N)$-recouvrement pour cette partie. Mais la mesure de $\bigcup_{x \in \mathcal{R}^{m}} B_{m N}(x, \epsilon) \times M$ pour $\mu$ est égale à celle de $\bigcup_{x \in \mathcal{R}^{m N}} B_{m N}(x, \epsilon)$ pour $\nu$, mesure minorée indépendamment de $m$ par définition de $\mathcal{R}^{m N}$. Donc $\mathcal{Q}$ est un recouvrement pour $(X \times M, F, \mu)$.

Il reste à majorer \# $\mathcal{Q}$. Pour ce faire, on associe à chaque $\left(x^{\prime}, y^{\prime}\right) \in \mathcal{Q}$, un $m+1$-uplet $\left(x, y_{0}, \ldots, y_{m-1}\right) \in \mathcal{T}^{m N}$ tel que $d_{m N}\left(x^{\prime}, x\right)<\delta<\frac{1}{2} \epsilon$ et $d\left(f_{x^{\prime}}^{k N+i} y, f_{x}^{i} y_{k}\right)<\frac{1}{2} \epsilon$. Remarquons que cette application $\mathcal{Q} \rightarrow \mathcal{T}^{m N}$ est injective. Pour conclure, il suffit de vérifier que tout $\left(x^{\prime}, y^{\prime}\right) \in \mathcal{Q}$ a une image bien définie.

Prenons d'abord $x \in \mathcal{R}^{m N}$ avec $d_{m N}\left(x, x^{\prime}\right)<\delta$. Soit $0 \leq k<m$. Comme $d_{N}\left(G^{k N} x, G^{k N} x^{\prime}\right)<\delta$, on a, pour $0 \leq i<N$,

$$
d\left(f_{x^{\prime}}^{i+k N} y^{\prime}, f_{G^{k N} x}^{i}\left(f_{x^{\prime}}^{k N} y^{\prime}\right)\right)<\frac{1}{10} \epsilon .
$$

TOME $126-1998-\mathrm{N}^{\circ} 1$ 
Maintenant il existe $y_{k} \in \mathcal{S}_{G^{k N} x}^{N}$ tel que

$$
d\left(f_{G^{k N} x}^{i}\left(y_{k}\right), f_{G^{k N} x}^{i}\left(f_{x^{\prime}}^{k N} y^{\prime}\right)\right)<\frac{1}{10} \epsilon
$$

pour $0 \leq i<N$. Ainsi :

$$
d\left(f_{x^{\prime}}^{k+i} y^{\prime}, f_{G^{k N} x}^{i} y_{k}\right)<\frac{1}{5} \epsilon, \quad \forall k, 0 \leq k<m, \forall i, 0 \leq i<N
$$

Donc $\left(x, y_{0}, \ldots, y_{m-1}\right)$ peut être associé à $\left(x^{\prime}, y^{\prime}\right)$. Ceci termine la preuve du lemme.

Preuve de la proposition. - Fixons $\mu$ une mesure de probabilité invariante et ergodique de $\left([0,1]^{d}, F\right)$ de grande entropie.

Soit $Q$ la partition naturelle pour $G$. D'après l'hypothèse de récurrence et le lemme ci-dessus, on peut supposer que pour $\pi \mu$-presque tout $x \in[0,1]^{d-1}:$

$$
\lim _{n \rightarrow \infty} \operatorname{diam}\left(Q^{n}(x)\right)=0 .
$$

Soit l'ensemble $B$ des points $x \in[0,1]^{d}$ tels que :

(1) $\Gamma(x) \stackrel{\text { def }}{=} A \in P^{\mathbb{N}}$ tel que $f^{n}(x) \in A_{n}$ pour tout $n \geq 0$ est bien défini ;

(1) $P^{\infty}(x) \stackrel{\text { def }}{=} \bigcap_{n \geq 0}\left[A_{0} \ldots A_{n}\right]$ n'est pas réduit à $x$ (ici $\left.A=\Gamma(x)\right)$;

(1) $Q^{\infty}(\pi(x))=\{\pi(x)\}$.

Cet ensemble $B$ est une partie invariante et mesurable de $[0,1]^{d}$.

Montrons que $\mu(B)=0$. Sinon, $\mu(B)=1$. Définissons :

$$
x \equiv y \Longleftrightarrow P^{\infty}(x)=P^{\infty}(y) \quad \text { pour } x, y \in B
$$

Remarquons que pour $x \in B, P^{\infty}(x)$ est un intervalle vertical non trivial. Donc tout intervalle vertical ne peut contenir qu'un nombre dénombrable d'ensembles distincts de la forme $P^{\infty}(x)$ avec $x \in B$. Ainsi, l'intersection de $B$ avec tout intervalle vertical est dénombrable après quotient par $\equiv$. Ceci veut dire que $\left([0,1]^{d}, F, \mu\right) / \equiv$ est une extension dénombrable de $\left([0,1]^{d-1}, G, \pi \mu\right)$. En particulier, ces deux systèmes ont la même entropie (prop. 2.8 de [6]). Cette entropie est donc majorée par $h_{\text {top }}(G) \leq h_{\text {top }}^{d-1}(F)<h_{\text {top }}(F):(F, \mu) / \equiv$ a petite entropie.

Montrons :

$$
h(F, \mu) \leq h((F, \mu) / \equiv) .
$$


Soit $H$ une partition de $[0,1]^{d}$ en $N$ bandes de la forme $[0,1]^{d-1} \times$ $\left[i / N,(i+1) / N\left[\right.\right.$. Comme $F^{n} \mid P^{\infty}(x)$ (où $x$ appartient à $B$ ) est monotone pour tout $n \geq 0$, il est facile de voir que le nombre d'éléments de $H^{n}$ qui rencontrent n'importe quel $P^{\infty}(x)$ fixé, avec $x \in B$, est majoré par $(N-1) n+1$. Soit $\epsilon_{0}>0$. Fixons $n_{0}$ si grand que $N n_{0} \leq e^{\epsilon_{0} n_{0}}$. Mettons un ordre total quelconque sur $H^{n_{0}}$. Soit $x \in B$; écrivons

$$
\left\{H^{n_{0}}(y) ; y \in P^{\infty}(x)\right\}=\left\{I_{1}(x)<\cdots<I_{r}(x)\right\}
$$

avec $1 \leq r<N n_{0}$. Définissons $\Phi(x)=k$ si $H^{n_{0}}(x)=I_{k}(x)$. Considérons l'application mesurable :

$$
\begin{aligned}
& B \longrightarrow[0,1]^{d} / \equiv \times \mathcal{S} \\
& x \longmapsto\left(P^{\infty}(x), 0^{n_{0}-1} \Phi(x) 0^{n_{0}-1} \Phi\left(F^{n_{0}} x\right) 0^{n_{0}-1} \cdots\right)
\end{aligned}
$$

où $\mathcal{S}$ est le sous-shift sur $\left\{0,1, \ldots, n_{0}\right\}$ défini en imposant aux positions occupées par un symbole non nul de différer d'un multiple de $n_{0}$. La dynamique sur l'image de $B$ est $F \times \sigma$, où $\sigma$ est le décalage. II est maintenant évident que, pour tout $\epsilon_{0}>0$,

$$
h(F, \mu, P \vee H) \leq h((F, \mu) / \equiv)+\epsilon_{0} .
$$

L'union de $P$ et des $H$ pour $N \geq 1$ sépare presque toutes les orbites. On obtient donc la majoration de $h(F, \mu)$ et (4.7) est démontrée.

Par conséquent, $\mu$ a une petite entropie. Cette contradiction montre que $\mu(B)=0$. Nous avons montré que pour tout $x \in[0,1]^{d}$ en dehors d'un ensemble $h$-négligeable :

$$
\bigcap_{n \geq 1} P^{n}(x)=\{x\}
$$

Il faut maintenant voir qu'on a un peu plus, à savoir :

$$
\lim _{n \rightarrow \infty} \operatorname{diam}\left(P^{n}(x)\right)=0 .
$$

Supposons par contradiction que $\operatorname{diam}\left(P^{n}(x)\right)>2 \epsilon$ pour tout $n \geq 1$, un $\epsilon>0$ et tout $x$ pris dans un ensemble de mesure positive.

D'après les propriétés des intersections d'intervalles emboîtés, il découle de l'égalité $P^{\infty}(x)=\{x\}$ que le diamètre des intervalles

$$
P^{n}(x) \cap(\{\pi(x)\} \times[0,1])
$$

TOME $126-1998-\mathrm{N}^{\circ} 1$ 
tend vers zéro quand $n$ tend vers $\infty$. Donc, pour presque tout $x$, il existe des entiers $n$ et $m$ tels que $F^{n}$ envoie $[x, x+(0, \ldots, 0, \epsilon)]$ par un homéomorphisme sur un intervalle contenant un point critique $c$ et de même pour $F^{m},[x-(0, \ldots, 0, \epsilon), x]$ et un point critique $d$. Les points critiques $c$ et $d$ appartiennent aux hypersurfaces critiques qui sont presque horizontales et s'étendent au-dessus de tout $[0,1]^{d-1}$. L'application $F$ est continue. Il existe donc $\delta>0$ (dépendant de $x$ ) telle que, pour tout $y \in B(x, \delta),\left.F^{n+1}\right|_{[y, y+(0, \ldots, 0, \epsilon)]}$ n'est pas un homéomorphisme et de même pour $\left.F^{m+1}\right|_{\mid[y-(0, \ldots, 0, \epsilon), y]}$. Par l'hypothèse de récurrence, il existe $N$ tel que

$$
Q^{N}(\pi(x)) \subset B(\pi(x), \delta) .
$$

En remplaçant $N$ par $\max (N, n, m)$, on obtient que $P^{N}(x) \subset B(x, \sqrt{2} \epsilon)$ (supposons que $\delta<\epsilon$ ). Comme $\sqrt{2} \epsilon<2 \epsilon$, c'est une contradiction. Ceci termine la preuve de la proposition.

\section{Conclusion}

Nous démontrons le théorème principal. Il suffit de vérifier les hypothèses des théorèmes 2.2 et 2.4 . Soient $f_{1}, \ldots, f_{d}$ comme dans le théorème principal. On peut supposer que $h_{\text {top }}\left(f_{1}\right) \geq \cdots \geq h_{\text {top }}\left(f_{d}\right)$.

Pour $\epsilon>0$ assez petit, le lemme 4.1 implique que $\left([0,1]^{d}, F\right)$ avec sa partition naturelle est inversible par morceaux et satisfait l'inégalité entropique (2.3) :

$$
h_{\text {top }}(\partial P, F)+h_{\text {mult }}(P, F)<h_{\text {top }}(F) .
$$

De plus, la proposition 4.2 dit que $P h$-sépare. Le théorème 2.2 s'applique : $F$ est isomorphe au sens de l'entropie à la chaîne de Markov topologique définie par son diagramme de Markov connexe.

Considérons le théorème 2.4. D'après la remarque après l'énoncé de ce théorème, il faut majorer uniformément le «nombre» de $(\epsilon, n)$-orbites issues de $\partial P$, multiplicité comprise, i.e., établir les majorations (2.5) et (2.6) pour des constantes $H_{1}+H_{2}<h_{\text {top }}(f)$. Rappelons la propriété de «quasi sous-multiplicativité» de $n \mapsto \sup _{\sigma} r(\epsilon, n, \operatorname{Im} \sigma)$ énoncée dans la proposition 3.10. Elle implique que nous avons l'estimation uniforme suivante : pour tout $H_{1}>H_{\text {top }}^{d-1}\left(F_{0}\right)$, pour tout $\epsilon>0$, il existe $C<\infty$ tel que pour $F C^{\infty}$-proche de $F_{0}$ :

$$
r(\epsilon, n, \partial P) \leq C \cdot \mathrm{e}^{H_{1} n}, \quad \forall n \geq 0 .
$$

C'est (2.5). 
Maintenant, la suite $n \mapsto \operatorname{mult}\left(P^{n}\right)$ est clairement sous-multiplicative. D'après la discussion de la section 4.1 , pour tout $H_{2}>0$, tout $F C^{\infty}$ proche de $F_{0}$ :

$$
\operatorname{mult}\left(P^{n}\right) \leq C^{\prime} \cdot \mathrm{e}^{H_{2} n}, \quad \forall n \geq 0
$$

C'est (2.6).

Comme $H_{\text {top }}^{d-1}(F)<h_{\text {top }}(F)$ pour $F$ proche de $F_{0}$, on peut bien obtenir que $H_{1}+H_{2}<h_{\text {top }}(F)$. Ainsi, $N$ (défini dans l'énoncé du théorème 2.4) peut être choisi uniformément pour $F$ dans un petit voisinage du produit direct $F_{0}$. Fixons un tel $N$.

Rappelons que chaque élément de $\mathcal{D}_{N}$ correspond à une composante connexe de $[0,1]^{d}$ divisée par les points critiques de $F^{k}, k \leq N$. Or, pour $F=F_{0}$, l'ensemble critique de $F^{k}$ est un quadrillage et $\mathcal{D}_{N}$ est donc fini. Ceci reste vrai par perturbation pour $k \leq N$ dans un voisinage de $F_{0}$ dépendant de $N$, qui est fixé. Le théorème 2.4 s'applique donc et implique la finitude du nombre de sous-chaînes irreductibles de grande entropie.

L'énoncé sur les mesures maximales s'en déduit aisément en utilisant les deux résultats non-triviaux suivants :

(1) Chaque sous-chaîne irréductible porte au plus une mesure maximale (voir B. Gurevič [10], [11]). Le théorème 2.4 donne qu'il y a un nombre fini de mesures maximales.

(1) Chaque application $C^{\infty}$ sur une variété compacte a au moins une mesure maximale (voir S.E. Newhouse [20]).

\section{BIBLIOGRAPHIE}

[1] Bowen (R.). - Entropy for group endomorphisms and homogeneous spaces, Trans. Amer. Math. Soc., t. 153, 1971, p. 401-414.

[2] Buzzi (J.). - Entropies et représentation markovienne des applications régulières de l'intervalle. - Thèse, Université Paris-Sud, Orsay, 1995 .

[3] Buzzi (J.). - Intrinsic Ergodicity of Smooth Interval Maps, Israel J. Math., t. 100, 1997, p. 125-161.

[4] Buzzi (J.). - Intrinsic Ergodicity of Affine Maps on $[0,1]^{d}$, Monat. Math., t. 124, 1997, p. 97-118.

TOME $126-1998-\mathrm{N}^{\circ} 1$ 
[5] Buzzi (J.). - Entropy, volume growth and Lyapunov exponents, submitted.

[6] Buzzi (J.). - Markov extensions for multi-dimensional dynamical systems, submitted.

[7] Coste (M.). - Ensembles semi-algébriques, Lecture Notes in Mathematics, $\mathrm{n}^{\circ}$ 959, Springer-Verlag, Berlin, 1982, p. 109-138.

[8] Denker (M.), Grillenberg (C.), Sigmund (K.). - Ergodic theory on compact spaces, Lecture Notes in Mathematics $n^{\circ} \mathbf{5 2 7}$, SpringerVerlag, $197^{6}$.

[9] Gromov (M.). - Entropy, homology and semi-algebraic geometry, Séminaire Bourbaki, t. 663, 1985-1986.

[10] Gurevič (B.M.). - Topological entropy of enumerable Markov chains, Soviet Math. Dokl., t. 10, 1969, p. 911-915.

[11] Gurevič (B.M.). - Shift entropy and Markov measures in the path space of a denumerable graph, Soviet Math. Dokl., t. 11, 1970, p. $744-747$.

[12] Hirsch (M.W.), Pugh (C.C.), Shub (M.). - Invariant Manifolds, Lecture Notes in Mathematics $n^{\circ}$ 583, Springer-Verlag, Berlin, 1977.

[13] Hofbauer (F.). - On intrinsic ergodicity of piecewise monotonic transformations with positive entropy, Israel J. Math., t. I, 34, 1979, p. 213-237; t. II, 38, 1981, p. 107-115.

[14] Kаток (A.). - Lyapunov exponents, entropy and periodic orbits for diffeomorphisms, Publ. Math. I.H.E.S., t. 51, 1980, p. 137-173.

[15] Keller (G.). - Markov extensions, zeta functions, and Fredholm theory for piecewise invertible dynamical systems, Trans. Amer. Math. Soc, t. 314, 1989, p. 433-497.

[16] Keller (G.). - Lifting measures to Markov extensions, Mh. Math., t. 108, 1989, p. 183-200.

[17] Ledrappier (F.), Walters (P.). - A relativised variational principle for continuous transformations, J. London Math. Soc., t. 16, 1977, p. $568-576$.

[18] Misiurewicz (M.). - Horseshoes for mappings of the interval, Bull. Acad. Polon. Sci., Ser. Sci. Math., t. 27, 1979, p. 167-169.

[19] Misiurewicz (M.), Szlenk (W.). - Entropy of piecewise monotone mappings, Studia Math., t. 67, p. 45-63.

[20] Newhouse (S.E.). - Continuity properties of the entropy, Ann. Math., t. 129, 1989, p. 215-237.

[21] Weiss (B.). - Intrinsically ergodic systems, Bull. Amer. Math. Soc., t. 76, 1970, p. 1266-1269.

[22] Yomdin (Y.). - Volume growth and entropy, Israel J. Math., t. 57, 1987 , p. $285-300$. 